Chapter 9

\title{
Extended Aortic Replacement Via Median Sternotomy with Left Anterolateral Thoracotomy
}

\author{
Satoshi Yamashiro, Yukio Kuniyoshi, \\ Hitoshi Inafuku, Yuya Kise and Ryoko Arakaki \\ Additional information is available at the end of the chapter \\ http://dx.doi.org/10.5772/50809
}

\section{Objective}

Prevention of cerebral injury is an important consideration during repair of an aortic arch aneurysm, and a major goal of cerebral protection techniques. We describe extended thoracic aortic aneurysms treated using our surgical strategy.

\section{Patients and methods}

Between January 2001 and December 2011, 31 patients (22 men and 9 women; mean age, of $68.9 \pm 9.5$ y) underwent total replacement of the arch and descending aorta. Seven patients underwent emergency surgery (22.6\%). At that time, a median sternotomy with left anterolateral thoracotomy provided a good visual field and bilateral axillary arteries were preferentially used for systemic as well as selective cerebral perfusion.

\section{Results}

Two patients died in hospital (hospital mortality, 6.5\%). Mechanical ventilation was required after surgery for $6.3 \pm 7.4$ days. Permanent neurological dysfunction developed in 1 ( $3.2 \%)$ patient who died of sepsis 2 years after the operation. Although prolonged mechanical ventilation support was necessary, all patients recovered uneventfully. 


\section{Conclusion}

Our results suggested that total arch replacement through a median sternotomy plus left anterolateral thoracotomy seems to be helpful for extended replacement of the thoracic aorta, as well as a distal re-operation for dissecting type A. Moreover, our results suggested that perfusion from the bilateral axillary arteries can help prevent cerebral damage.

Despite advances in surgical techniques such as management of anesthesia and cardiopulmonary bypass (CPB)[1,5], brain injury after aortic arch surgery remains an important source of morbidity and mortality because of the advanced age of the patients and the presence of severe comorbidities [6]. Extended thoracic aortic aneurysms involving the ascending aorta, arch, and descending aorta are often approached in staged operations [7]. However, a single-stage replacement of the aortic arch and descending aorta might be a preferable surgical option for specific types of atherosclerotic aneurysm, such as chronic type A or B dissection. We describe our experience with extended aortic replacement through a median sternotomy with left anterolateral thoracotomy using our current strategy.

\section{Patients and methods}

Between January 2001 and December 2011, 31 consecutive patients with extended thoracic aortic aneurysms underwent total replacement of the arch and descending aorta at our institution.

\begin{tabular}{ll}
\hline Total number of patients & 31 \\
\hline Age & $68.9+/-9.5$ years $(56-83)$ \\
\hline Gender (M/F) & $22 / 9$ \\
\hline Diagnosis & $11(35.5 \%)$ \\
\hline Atherosclerotic & $7(22.6 \%)$ \\
\hline Chronic type B dissection & $7(22.6 \%)$ \\
\hline Chronic type A dissection & $3(9.7 \%)$ \\
\hline Acute type A dissection & $3(9.7 \%)$ \\
\hline Infectious & $6(19.4 \%)$ \\
\hline Emergency & $3(9.7 \%)$ \\
\hline Rupture (impending) & $3(9.7 \%)$ \\
\hline Acute type A dissection & $9(34.8 \%)$ \\
\hline Previous operative history & $7(22.6 \%)$ \\
\hline Ascending aorta or Arch replacement & $1(3.2 \%)$ \\
\hline AVR + CABG & $1(3.2 \%)$ \\
\hline CABG & $2(6.5 \%)$ \\
\hline Y graft replacement for AAA &
\end{tabular}




\begin{tabular}{ll}
\hline Total number of patients & 31 \\
\hline Underlying dysorders & \\
\hline HT & $23(74.2 \%)$ \\
\hline DM & $11(35.5 \%)$ \\
\hline HL & $18(58.1 \%)$ \\
\hline Concomitant operation & $5(16.1 \%)$ \\
\hline AVR & $2(6.5 \%)$ \\
\hline AVR+MVR+CABG & $1(3.2 \%)$ \\
\hline Bentall operation with CABG & $1(3.2 \%)$ \\
\hline
\end{tabular}

Table 1. Characteristics of patients who underwent total arch with descending aortic replacement for extended thoracic aortic aneurysm between January 2001 and December 2011 at our institution. M, male; F, female; AVR, aortic valve replacement; CABG, coronary artery bypass grafting; AAA, abdominal aortic aneurysm; HT, hypertension; DM, diabetes mellitus; $\mathrm{HL}$, hyperlipidemia; MVR, mitral valve replacement.

Table 1 shows the characteristics of the 22 male and 9 female patients (mean age, of $68.9 \pm 9.5$ y; range, 56 to $83 \mathrm{y}$ ). Eleven had atherosclerotic distal arch aneurysms, 7 had chronic type B dissection, 7 had chronic type A dissection after graft replacement of the ascending aorta or aortic arch, 3 had acute type A dissection, and 3 had infectious arch aneurysms. Three patients had a ruptured or impending ruptured aneurysm of the arch aorta, and three patients had acute type A dissection that required emergency surgery (22.6\%). Coronary angiography was also performed before surgery for all patients except for those in emergency. Seven patients had undergone a previous graft replacement of the ascending aorta, 1 had aortic valve replacement (AVR), and 1 had a coronary artery bypass graft (CABG). Five patients underwent concomitant cardiac procedures, namely CABG in 2, AVR in 1, AVR and mitral valve replacement (MVR) with CABG in 1 and the Bentall procedure with CABG in 1 .

\section{Surgical technique}

Patients were endotracheally intubated with a double-lumen tube to deflate the lungs and then positioned on an operating table with the chest rotated 60 degrees from supine towards the right. A 5- to 6-cm transverse skin incision was made before the median sternotomy at about $1 \mathrm{~cm}$ below the middle and lateral part of the clavicle. The axillary and right femoral arteries (FA) were exposed, and the heart, ascending aorta, aortic arch, and arch vessels were exposed through a median sternotomy with a sternum transection plus a left fourth intercostal thoracotomy (the "Door open" method). This combined approach was applied if the predicted level of the distal anastomosis was below the sixth vertebra, and it provided a good visual field of the whole heart as well as of the entire aorta. The perfusion site was selected based on preoperative computed tomographic scans. Bilateral or right axillary arteries (bilateral in 17, right in 13) with or without the right FA were used as the perfusion site for all except 1 patient for whom the ascending aorta was used with the right femoral artery. 
When using the axillary artery, an 8-mm-diameter graft was anastomosed to the bilateral or right axillary arteries for systemic arterial cannulation after systemic heparinization. These grafts were used for antegrade selective cerebral perfusion (SCP) after deep hypothermic circulatory arrest (HCA). Antegrade SCP was established through vascular grafts anastomosed to the bilateral axillary arteries and a perfusion catheter placed directly into the left carotid artery. Moreover, the left common carotid artery was directly cannulated as described [9], to prevent cerebral thromboembolization in two patients. Figure 1 shows a schema of the surgical approach and cerebral protection. Distal anastomosis proceeded under crossclamping of the distal descending aorta with distal perfusion through FA in 18 patients to prevent visceral ischemia. The remaining 14 patients underwent open distal anastomosis, after the completion of which, the graft was drawn anteriorly into the isolated residual distal arch aorta. Meanwhile the left phrenic and left recurrent laryngeal nerves were identified, mobilized with the aneurysmal wall, and protected. Antegrade SCP was terminated after reconstruction of the arch vessels, and then the proximal side of arch graft was sutured to the stump of the ascending aorta. The root of the left subclavian artery was ligated when an 8mm-diameter graft was anastomosed to the left axillary artery, and reconstructed by graftgraft anastomosis using a 5-0 polypropylene running suture.
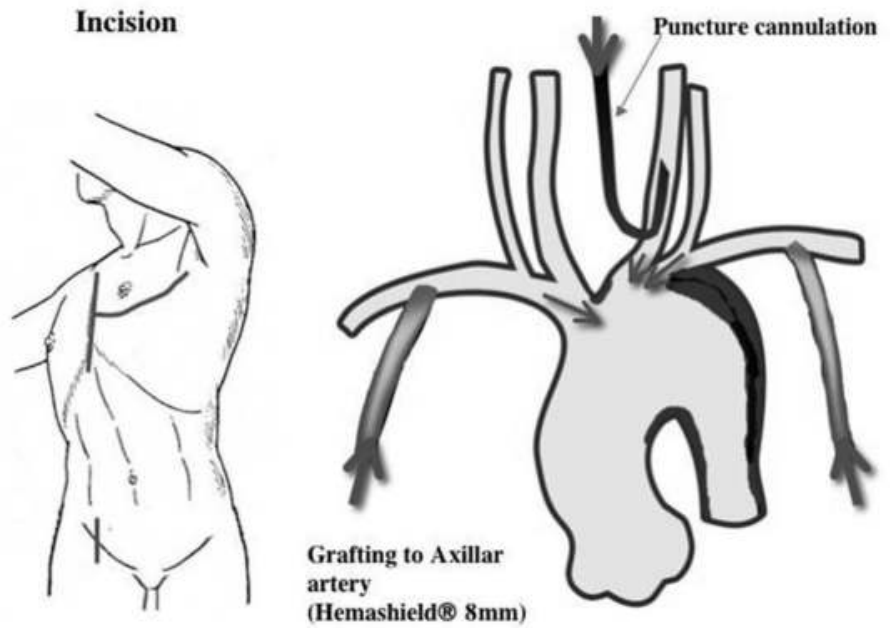

Figure 1. Schema of surgical approach and cerebral protection.

\section{Results}

Table 2 shows that the durations of surgery, total cardiopulmonary bypass (CPB), cardiac ischemia, HCA and SCP were 669.6 \pm 191.7, $247.9 \pm 79.6,163.0 \pm 71.3,55.6 \pm 20.9$ and 117.4 
\pm 32.5 minutes, respectively. None of these values significantly differed between patients after total arch replacement through a median sternotomy and those who also had a left anterolateral thoracotomy (data not shown).

\begin{tabular}{ll}
\hline Surgical duration & $669.6+/-191.7$ minutes \\
\hline Total CPB time & $247.9+/-79.6$ minutes \\
\hline Cardiac arrest time & $163.0+/-71.3$ minutes \\
\hline SCP time & $117.4+/-32.5$ minutes \\
\hline HCA time (13 patients) & $55.6+/-20.9$ minutes \\
\hline Hospital death & $2(6.5 \%)$ \\
\hline Mechanical ventilation duration & $6.3+/-7.4$ days \\
\hline Complication & $22(71.0 \%)$ \\
\hline Respiratory failure & $1(3.2 \%)$ \\
\hline Cerebral infarction & $2(6.5 \%)$ \\
\hline Acute renal failure & $35.3+/-6.7$ days \\
\hline Hospitalization after surgery & $1(3.3 \%)$ \\
\hline Fate death & $58.1+/-26.1$ months \\
\hline
\end{tabular}

Table 2. Operative results. CPB, cardiopulmonary bypass; SCP, selective cerebral perfusion; HCA, hypothermic circulatory arrest.

Two patients died in hospital (hospital mortality, 6.5\%). One patient with a ruptured arch aneurysm died of multiple organ failure (MOF) on postoperative day 70 . He had undergone emergency surgery due to aneurysmal rupture and was in a state of preoperative shock. The other patient had a distal pseudoaneurysm after arch replacement for type A dissection, and required simultaneous AVR and MVR with CABG. He died of MOF on postoperative day 17. However, neither of these patients was complicated with cerebral damage.

Early morbidity in 22 patients comprised pulmonary failure, defined as requiring respiratory support for $>48$ hours. Mechanical ventilation was required after surgery for $6.3 \pm 7.4$ days. Two patients who had preoperative left recurrent laryngeal nerve palsy developed persistent hoarseness after the operation. No new phrenic or left recurrent laryngeal nerve palsies developed as a result of surgery. Permanent neurological dysfunction, defined as permanent neurological deficits with localizing neurological signs and corresponding new defects on CT images, occurred in $1(3.2 \%)$ patient, who died of sepsis 2 years after the operation. The length of the hospital stay after surgery was $35.3 \pm 6.7$ days. The actuarial survival rate at 5 years after surgery was $90.1 \%$ (Figure 2), and the patients were followed up after surgery for $58.1 \pm 26.1$ months. 


\section{Actuarial survival rate}

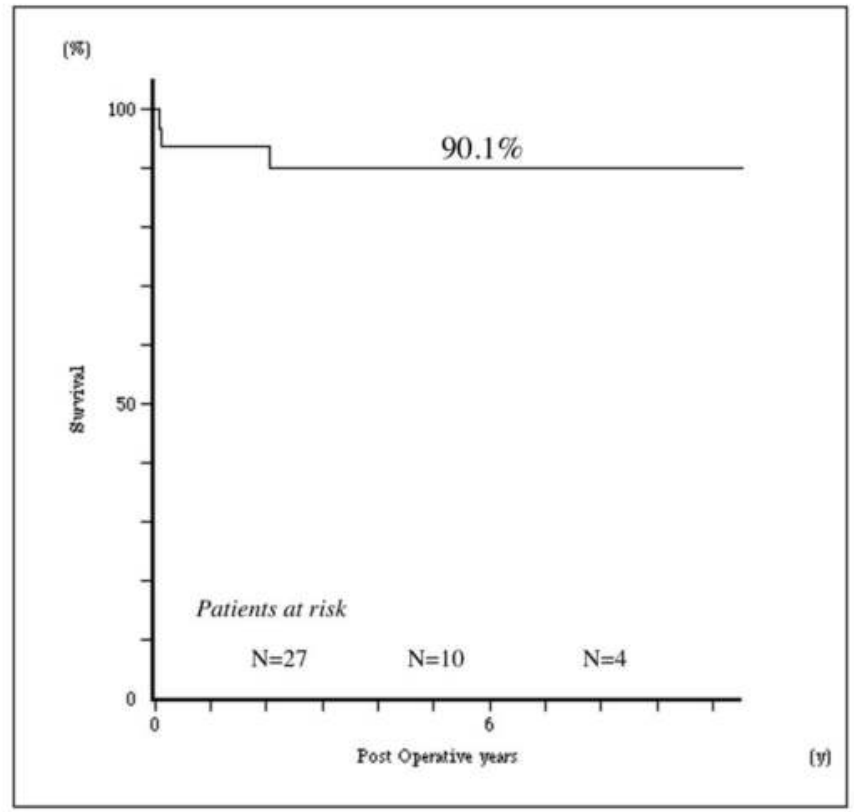

Figure 2. Actuarial survival rates among 31 patients with extensive replacement of the thoracic aorta.

\section{Discussion}

Indications for approaches in patients with arch aneurysms are controversial, because the surgical results of extended arch replacement are suboptimal. The combined surgical approach (median sternotomy with left anterolateral thoracotomy) to treating thoracic aortic aneurysms has been considered too invasive [3]. We found in our series that the durations of surgery and of relative mechanical circulation were significantly elongated. Therefore, 17 patients required respiratory support for $>72 \mathrm{~h}$, although they gradually recovered without complications. Mechanical ventilation was required after surgery for $7.3 \pm 8.4$ days. All of the surviving patients recovered uneventfully except for those who became complicated with cerebral infarction. The combined approach seemed to be too invasive for respiratory function. On the other hand, Ohata et al. [2] found no significant differences in respiratory function or in the inflammatory response between patients who underwent total arch replacement through a median sternotomy and those who also had a left anterolateral thoracotomy. Especially in the distal reoperative situation for dissecting type A, the retrosternal space can be easily excised from a left thoracotomy, although adhesion due to previous surgeries can be moderate or severe. The cannulae are conveniently situated anteriorly away from the main operative field, which improves access to the aortic arch and descending thoracic aorta. Moreover, the ability to 
visualize and protect the phrenic and recurrent laryngeal nerves contributes to extending the recovery of patients with impaired respiratory function.

The prevention of cerebral embolism is an important consideration during repair of an aortic arch or ascending aortic aneurysm, and is a major goal of cerebral protection techniques. Selection of the arterial cannulation site for $\mathrm{CPB}$ is critical o achieve this. The ascending aorta or FA is usually selected as an arterial cannulation site $[1,5]$. However, cerebral embolism can occur in both cannulation sites, because of severe atherosclerotic changes in the ascending aorta near the arch aneurysm or retrograde perfusion via the femoral artery $[3,11]$. The important variables that are considered to influence the occurrence of postoperative stroke following aortic arch repair, and which might be affected by the method of cannulation, include the presence of clots and atheromas in the aorta, and dissection as the etiology of aneurysms. Svenson et al. [8] started to use subclavian or axillary artery cannulation strategy with HCA and antegrade brain perfusion 1995, and found it to be a safe approach for aortic arch surgery as the stroke risk was $<2 \%$. The theoretical advantages of using the subclavian or axillary artery site for inflow for complex cardiac and cardioaortic operations have recently become apparent $[8,9]$. These possible advantages include a decreased likelihood of stroke from embolic material, less likely malperfusion with aortic dissection, reduced disruption of atheroma or calcified plaques, and the ability to administer antegrade brain perfusion. Only one patient in our series who had undergone ascending aortic perfusion developed cerebral infarction. We suspected that the infarction was due to embolism from an atheroma in the arch aorta. Since 2000, we have preferentially used axillary arteries for perfusion with total arch replacement except for this patient. No other patients have developed neurologic injury since then.

Ergin et al. [10] reported that temporary neurological dysfunction was a clinical marker of an insidious but significant neurological injury associated with measurable long-term deficits in cerebral function. Therefore, we have used only SCP in aortic arch repair because of the extended duration of cerebral safety and the low incidence of temporary neurological dysfunction. However, atheromatous emboli traveling to the brain, which is considered to be a main cause of permanent neurological dysfunction, remains a serious major concern during SCP. Antegrade SCP is physiological and the time taken to ensure cerebral safety should be much longer. However, arch-vessel cannulation is required for this maneuver, which carries a risk of cerebral embolization. Many embolic sources are caused by systemic $\mathrm{CPB}$ perfusion via the ascending aorta across the arch aneurysm, or otherwise, retrograde $\mathrm{CPB}$ perfusion via the FA, or cannulation to the arch vessels for SCP. The need to cannulate arterial vessels and to manipulate often severely atherosclerotic aneurysms enhances the potential for embolization into the cerebral circulation, resulting in focal lesions and neurological injury. Svenson and colleague [9] described that when using the subclavian or axillary arteries for inflow, direct cannulation is associated with a greater risk of local complications, including dissection of the artery, inadequate flow, abutment of the cannula tip against the carotid artery wall, and tears that are difficult to repair because arterial tissue is fragile and often traumatized. We then switched arterial inflow to a side graft sewn to the vessels, and a delicate and sometimes difficult repair of the artery was not required at the end of the operation. Instead, the side graft could simply be over-sewn and tied off or clipped. We doubt 
whether whole brain perfusion is sufficient with only right axillary artery perfusion. The best approach for cerebral protection during these procedures remains a matter of controversy. Although the procedures are continually undergoing refinement with improved results, associated brain injury can still arise despite the application of all cerebral protection techniques suitable for these operations. Thus, left-side brain perfusion was added. Therefore, bilateral axillaries were routinely used as the perfusion site in current 17 patients. Moreover, vertebral perfusion via the left axillary artery is important for spinal as well as cerebral protection. Simultaneously sewing a graft to a bilateral axillary artery takes about 20 to 30 minutes. The axillary artery always has less atherosclerotic change than the ascending aorta or the FA, and it can easily be exposed [12-14]. During reconstruction of the left subclavian artery, a side graft could simply be sewn onto a graft that was anastomosed to the left axillary artery beforehand.

Recently, several reports of total endovascular treatment of aortic arch with branched stent graft or fenestrated device demonstrated feasible results [15]. Kawaguchi et al [16] described that thoracic endovascular repair (TEVAR) tends to have a lower rate of serious complications than open surgery. They reported that cerebral infarction rate in patients who received a fenestrated device was 5.5\%. Actually, TEVAR does not require extracorporeal circulation; therefore, patients do not have low cerebral perfusion during the procedure. Certainly, we agree to endovascular treatment of pathologies affecting the ascending aorta and aortic arch is feasible in limited patients. Although, TEVAR cannot be considered a well-established treatment method just yet, especially in aortic arch. Technical difficulties with graft design and deployment persist [17]. Therefore, we thought extensive surgery have not been avoidable in this series, especially in dissection and patients who required concomitant procedures.

We believe that extensive reimplantation of the thoracic aorta accompanied by adequate distal aortic perfusion under distal clamping to avoid hypothermic circulatory arrest are effective. Our results suggested that total arch replacement through a median sternotomy plus left anterolateral thoracotomy allowed expeditious and extended replacement of the thoracic aorta and distal re-operation for dissecting type A aneurysms. This experience with median sternotomy together with left anterolateral thoracotomy approach and cannulation illustrates that our method is safe and effective. This study is limited by the relatively small size. However the analysis of our results may have practical implications in going evolution in these severe cases.

\section{Author details}

Satoshi Yamashiro*, Yukio Kuniyoshi, Hitoshi Inafuku, Yuya Kise and Ryoko Arakaki

*Address all correspondence to: y3104@med.u-ryukyu.ac.jp

Department of Thoracic and Cardiovascular Surgery, Graduate school of Medicine, University of the Ryukyus, Okinawa, Japan 


\section{References}

[1] Crawford, E. S., Kirklin, J. W., Naftel, D. C., Svensson, L. G., Coselli, J. S., \& Safi, H. J. (1992). Surgery for acute dissection of ascending aorta: should the arch be included? J Thorac Cardiovasc Surg, 104, 46-59.

[2] Kuniyoshi, Y., Koja, K., Miyagi, K., Uezu, T., Yamashiro, S., Arakaki, K., Mabuni, K., \& Senaha, S. (2004). Direct cannulation of the common carotid artery during the ascending aortic or aortic arch replacement. Jpn J Thorac Cardiovasc Surg, 52, 247-253.

[3] Wesatby, S., \& Katsumata, T. (1998). Proximal aortic perfusion for complex arch and descending aortic disease. J Thorac Cardiovasc Surg, 115, 162-167.

[4] Ohata, T., Sakakibara, T., Takano, H., \& Ishizaka, T. (2003). Total arch replacement for thoracic aortic aneurysm via median sternotomy with or without left anterolateral thoracotomy. Ann Thorac Surg, 75, 1792-1796.

[5] Kazui, T., Washiyama, N., Bashar, A. H. M., Terada, H., Suzuki, T., Ohkura, K., \& Yamashita, K. (2002). Surgical outcome of acute type A aortic dissection: analysis of risk factors. Ann Thorac Surg, 74, 75-82.

[6] Okita, Y., Ando, M., Minatoya, K., Kitamura, S., Takamoto, S., \& Nakajima, N. (1999). Predictive factors for mortality and cerebral complications in atherosclerotic aneurysm of the aortic arch. Ann Thorac Surg, 67, 72-78.

[7] Borst, H. G., \& Frank, G. (1988). Treatment of extensive aortic aneurysms by a new multiple-stage approach. J Thorac Surg, 95, 11-13.

[8] Svenson, L. G., Nadolny, E. M., Penney, D. L., Jacobson, J., Kimmel, W. A., Entrup, M. H., \& D'Agostino, R. S. (2001). Prospective randomized neurocognitive and S-100 study of hypothermic circulatory arrest, retrograde brain perfusion, and antegrade brain perfusion for aortic arch operations. Ann Thorac Surg, 71, 1905-1912.

[9] Svenson, L. G., Blackstone, E. H., Rajeswaran, J., Sabik, J. F., Lytle, B. W., GonzalezStawinski, G., Varvitsiotis, P., Banbury, M. K., Mc Carthy, P. M., Pettersson, G. B., \& Cosgrove, D. M. (2004). Does the arterial cannulation site for circulatory arrest influence stroke risk? Ann Thorac Surg, 78, 1274-1284.

[10] Ergin, M. A., Uysal, S., Reich, D. L., Apaydin, A., Lansman, S. L., Mc Cullough, J. N., \& Griepp, R. B. (1999). Temporary neurological dysfunction after deep hypothermic circulatory arrest: a clinical marker of long term functional deficit. Ann Thorac Surg, 67, 1887-1890.

[11] Numata, S., Ogino, H., Sasaki, H., Hanafusa, Y., Hirata, M., Ando, M., \& Kitamura, S. (2003). Total arch replacement using antegrade selective cerebral perfusion with right axillary artery perfusion. Eur J Cardiothorac Surg, 23, 771-775. 
[12] Strauch, J. T., Spielvogel, D., Lauten, A., Lansman, S. L., Mc Murtry, K., \& Bodian, Griepp. R. B. (2004). Axillary artery cannulation: routine use in ascending aorta and aortic arch replacement. Ann Thorac Surg, 78, 103-108.

[13] Kurisu, K., Ochiai, Y., Hisahara, M., Tanaka, K., Onzuka, T., \& Tominaga, R. (2006). Bilateral axillary arterial perfusion in surgery on thoracic aorta. Asian Cardiovasc Thorac Ann, 14, 145-149.

[14] Shimazaki, Y., Watanabe, T., Uchida, T., Takeda, F., Uesho, K., Koshika, M., Nakashima, K., \& Inui, K. (2003). Outcome of aortic arch surgery in patients aged 70 years or older: axillary artery cannulation and selective cerebral perfusion supports. J Cardiol, $41,7-12$.

[15] Melissano, G., Civilini, E., Bertoglio, L., Calliari, F., Setacci, F., Calori, G., \& Chiesa, R. (2007). Results of endografting of the aortic arch in different landing zones. Euro J Vasc Endovasc Surg, 33, 561-566.

[16] Kawaguchi, S., Yokoi, Y., Shimazaki, T., Koide, K., Matsumoto, M., \& Shigematsu, H. (2008). Thoracic endovascular aneurysm repair in Japan: Experience with fenestrated stent grafts in the treatment of distal arch aneurysms. J Vasc Surg, 48, 24S-29S.

[17] Brar, R., Ali, T., Morgan, R., Laftus, I., \& Thompson, M. (2008). Endovascular repair of an aortic arch aneurysm using a branched-stent graft. Eur J Vasc Endovasc Surg, 36, 545-549. 